\title{
Tendon damage in gout-a role for MSU crystals?
}

New research demonstrates that monosodium urate (MSU) crystals directly interact with tenocytes-reducing both cell viability and function-suggesting that these crystals can have negative affects on tendon tissues, which in turn might contribute to the development of damage to tendons in patients with gout.

"Although tendon damage is not as clinically apparent as cartilage or bone damage in patients with gout, recent

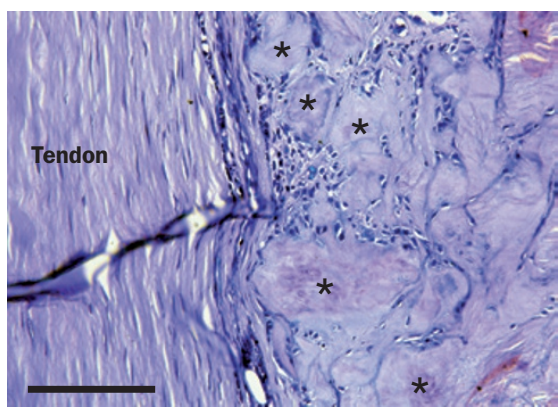

MSU crystals in contact with tendon affected by gout. Scale bar, $300 \mu \mathrm{m}$. *Crystalline core of the tophus. Image courtesy of A. Chhana. dual-energy CT and ultrasonography studies have demonstrated that tendons are frequently affected in gout," says author Ashika Chhana. As such, the investigators wanted to explore the role of tenocytes (the main cell type in tendons) in the development of tendon damage in gout.

Chhana and colleagues obtained surgical and cadaveric joint samples from patients with gout. Using histological and microscopy techniques, the researchers confirmed that, in the context of gout, MSU crystals were in direct contact with tenocytes and were found within, adjacent to and invading tendons as well as at the tendon-bone surface in joint samples.

The effects of MSU crystals on tenocyte viability and function in vitro were examined in both primary rat and human tenocytes. After $24 \mathrm{~h}, \mathrm{MSU}$ crystals reduced tenocyte viability in a dose-dependent manner, but did not markedly alter levels of tenocyte apoptosis. Moreover, real-time PCR analysis revealed that mRNA expression of tendon collagens, matrix proteins and degradative enzymes were reduced when tenocytes were cultured with MSU crystals. Collagen deposition by tenocytes was also reduced when MSU crystals were present.

"In the continued presence of MSU crystals, the healing of tendon in patients with gout will be limited, as the cells needed to initiate and drive the repair processes will continue to have impaired function," explains Chhana. She adds that effective therapies for tendon disease in gout should focus on dissolution of these MSU crystals to halt the progression of damage and enable subsequent recovery of the tenocyte cell population.

Katrina Ray

Original article Chhana, A. et al. Interactions between tenocytes and monosodium urate monohydrate crystals: implications for tendon involvement in gout. Ann. Rheum. Dis. doi:10.1136/annrheumdis-2013-204657 